\title{
Present status of total hip replacement
}

\author{
JOHN CHARNLEY \\ Wrightington Hospital, Wigan, Lancs.
}

In this discussion of the current status of total hip replacement I shall try to avoid technical details and concentrate on those aspects which will be most interesting to this distinguished body of physicians, which I am deeply honoured in being invited to address.

It is impossible to start without some elementary matters and first of all, one might ask 'What is total hip replacement?' The implants which have been put into the hip joint during the last $\mathbf{4 0}$ years have all been partial hip replacements, replacing either the femoral head or, as in the case of the SmithPeterson cup, the acetabulum. Total hip replacement means replacing the socket and the head at the same time.

Most of the successful total hip replacements are fixed into the body by means of a quick-setting plastic-acrylic-cement-though some surgeons attempt to avoid using cement. I do not have time to discuss this here though it is one of the most important aspects of total hip replacement; it is obviously futile to use a total hip implant if pain then originates from movement of the implanted elements on the bone.

The type of implant in which I am interested uses a small diameter metal head and a plastic socket of high density polyethylene. The small head is essential in the concept of what I call the 'low friction arthroplasty', or the 'low frictional torque arthroplasty', because the resistance to motion is lower with a small than with a large diameter head. The interior of one of these joints after $2 \frac{1}{2}$ years of service in the body shows a remarkably normal appearance of the tissues; there is no staining by metal particles and the appearances are astonishingly like those of a living joint.

After operation the patient gets out of bed usually on the 4th or 5th day, and starts to walk with full weight on the limb on the 7th day, depending of course on his age and agility. Most patients are fit to return home between $2 \frac{1}{2}$ and 3 weeks after the operation. Young patients have to be positively restrained for 4 to 6 weeks below their capacity for activity. Elderly patients, if they were able to live alone before the operation, will be able to go back to the same circumstances within 4 weeks. No physiotherapy and no attendances at hospital for outpatient treatment are needed after leaving hospital. Physiotherapy is prohibited after the patient leaves hospital lest these patients overdo it. The simple act of walking, or attempting to walk, eventually discarding all walking aids according to the patient's own common sense, is all that is needed in rehabilitation. The first attendance at hospital which is usually made 6 months after the operation is for psychological or social reasons; by that time the patient is almost completely rehabilitated.

The optimism which prompts these patients to return home early, or to be glad to go home, is quite different from what we used to see with patients after other types of hip operations; it comes from the fact that they know from their own sensation that the hip is going to be good. This optimism brings a new lease of life and it often works a profound change in their mental attitude, their general appearance at follow-up after surgery being enormously rewarding both for the surgical and nursing teams.

There is no special mortality after this operation, if we exclude massive fatal pulmonary embolism (not unique to this operation), which rates at about 0.5 to 1 per cent. The post-operative death rate from other causes reflects the risks which the surgeon is prepared to accept in the pre-operative state of the patient, even patients who have been taking cortisone for a long time can be effectively protected by a preoperative and post-operative prophylactic regime.

Deep wound infection is a serious post-operative hazard, but is not a hazard to life. Extreme precautions in the operating theatre are revealing themselves to be valuable in reducing this incidence of infection.

\section{Late results}

Since November, 1962, we have performed at Wrightington approximately 5,500 total hip re- 
placements, not including another 300 prototype operations carried out before 1962 using Teflon as the plastio for the socket. All the essential mechanical details, other than the actual nature of the plastic, were worked out before 1962 and since that time there have been no radical changes.

The present rate at Wrightington is about 1,200 operations a year, which makes it impossible to follow all recent patients beyond one year. We see patients 6 months after the operation and then after one year, and unless the case has special interest we then say goodbye to them. On the other hand, I maintain as a permanent study the 580 patients who were operated on between November, 1962, and December, 1965, and I hope to carry this on as long as is possible. Most of these early patients attend annually for examination, because most of them live locally; those who cannot attend, because of distance or physical frailty, are followed every year by questionnaires sent out by secretaries specially detailed for this work, and through the general practitioners we receive $x$ rays. By this special longterm study of what I call the 'First 500' I hope to get warning of any late defects which would not be detected by the routine following of large numbers of cases only for 2 to 3 years.

We are encountering an astonishing freedom from late defects in the 6 to 7 year results. The total death rate was approximately 2 per cent. (1.4 per cent. being deaths from pulmonary embolism). Mechanical failure occurred in no more than 1.5 per cent. and sepsis in about 3.5 per cent. In round figures (including deaths), 7 per cent. were not successful, which means a 93 per cent. success rate. I think we have now improved this to 95 per cent., but one must never forget that though 95 per cent. are successful, one patient in twenty will fail, and we always have to keep this in mind in recommending this operation.

\section{Indications and contraindications}

The indications for this operation are so wide that it will be most useful to discuss the contraindications.

\section{OSTEOARTHROSIS OF THE HIP JOINT}

This is numerically the largest group, and it must be emphasized as a general principle that total hip replacement should not be performed prophylactically. Whenever patients present for the first time with osteoarthrosis, and especially when they are in the 40 to 50-year age group, it is unwise to pontificate on the state of the hip without treatment in the next 4 to 5 years. One can usually be sure that it is going to be worse in 5 to 10 years, but I warn against predicting what is going to happen in 4 to 5 years. At the root of many disappointments, and even tragedies, after hip surgery in this age group there is an inadequate comprehension of the natural history of osteoarthrosis of the hip joint. A patient's fear of becoming totally disabled, often when there is a family history, can be magnified by the attitude of his physician. Some practitioners react to a diagnosis of arthrosis of the hip just as their patients do, so increasing the state of panic. Patients are often advised to try to avoid all mechanical strains in the hope of postponing the day when the joint will wear out completely, as though the hip joint in arthrosis were no more than a mechanical bearing, which it is not. Patients are often told such things as 'if you can sit, don't stand; if you can ride, don't walk'. But the facts are quite the opposite: patients who struggle to keep going and who exercise the arthrosic joint, despite pain, quite often will retain a surprising degree of function and mobility even in the presence of advanced radiological changes. I emphasize that I am speaking now of the $\mathbf{4 0}$ to $\mathbf{5 0}$-year olds. Nobody is a greater testimony to this general truth than my orthopaedic colleague, Mr. William Tucker, not only for his teaching in this field but as a personal example in his own hip joints.

My experience in patients reporting for the first time with arthrosis of the hip joint between 40 and 50 years of age is that, even without treatment, three out of four will be unchanged in 4 or 5 years, but one out of four will have degenerated within a year, with the joint space and head of the femur being destroyed.

Many patients come for an opinion on their arthrosic hip joints while they can still play nine holes of golf; I have not infrequently met patients of this type who have never played golf again after the operation of prophylactic osteotomy. I have known patients lose important jobs because of the failure of prophylactic osteotomy. These are the cases which degenerate rapidly and are not influenced by osteotomy. According to this cynical theory the three out of four who apparently benefitted by osteotomy would not have degenerated in any case!

When total hip replacement is being considered in patients in the younger age groups, with only moderate degrees of arthrosis, it is important to pose two questions:

'Are you coming for treatment for the state of your hip as it is now or are you merely anxious to avoid the possibility of being disabled in years to come, and of being told that you should have come earlier?'

and

'If you were never to get worse than this, would you be happy to carry on for some years without an operation?'

Usually the answers to these questions are unequivocal in deciding on postponement of surgery.

If a patient is unable to earn his living by reason 
of the state of his hips, then surgery is usually indicated even in young patients.

In persuading patients in the $40 \mathrm{~s}$ and $50 \mathrm{~s}$ to postpone surgery, if the condition is not truly disabling, it is important to dispel the false idea that a better result will be obtained by having total hip replacement early rather than late. No arthrosic hip joint can become too bad for total hip replacement. We must never forget that the operation of total hip replacement in 1971 is still only about 8 years old, and that not many surgeons have had experience of it for more than about 3 years. Nothing can be lost and much is to be gained in younger patients by waiting. We shall still make a few technical improvements. It is probable that by 1975,4 years from now, the extreme caution of these remarks will not be needed. When our knowledge of the late results is complete then, and then only, shall we be able to use total hip replacement when subjective symptoms seem to be out of proportion to objective findings.

In cautioning against performing total hip replacements, I am often challenged to define what type of failure I expect after many years. This is very difficult. So far I have encountered nothing to suggest that the products of wear or abrasion from the polyethylene socket will produce significant tissue reactions. To change worn sockets will be no great technical problem, because we worked all this out in changing Teflon sockets and the present operation is designed with this possibility in mind. Our excellent results after 7 years make it unlikely that further loosening of cement would occur by the further passage of time-most mechanical loosenings probably occur within 3 years. Nevertheless, most of our long-term results concern patients in the older age groups, and young patients will subject the bond between bone and cement to much greater violence, over much longer periods of time, than anything we have so far tested. This, therefore, is a field in which we must still be cautious, and not let ourselves be deterred by fear of future failures from the excessive optimism engendered by our present results.

In elderly patients great relaxation of caution is possible because every 5 years sees a reduction in the physioal demands on the hip joint. The only contraindioation to total hip replacement in such patients is unfitness for major surgery of any kind. As recently as 1967 , which is only 4 years ago, I was averse to total hip replacement in patients under 65 years of age unless they were grossly disabled, but to-day I routinely accept patients as young as 60 with only moderate disability if their subjective reaction appears to be genuine.

This brings us to a consideration of the psychiatric status of patients in relation to total hip replacement. It is one of the astonishing things about this operation that it can be used successfully in patients with an hysterical or grossly neurotic element in their personality. Patients with this temperament were often failures after the standard hip operations of the past, because such procedures often only mitigated pain and did not abolish it entirely. The hysteric is not interested in improvement, he wants absolute cure. But total hip replacement abolishes pain in at least 90 per cent. of cases and because of this absence of pain the operation is highly successful. In the senile patient, also, the threshold for discomfort is often at a childlike level, and the absence of pain after total hip replacement prevents the development of delayed surgical shock in these old people.

\section{OTHER CONDITIONS}

As regards total hip replacement in diseases other than osteoarthrosis, there are only two conditions where I think it is contraindicated. The first is the obvious one of suppurative or tuberculous arthritis, unless many years have elapsed. The second is the Charcot joint of tabes dorsalis. My solitary experience with this latter condition was with a case in which the hip dislocated after 6 months, and the dislocation became recurrent because of the absence of pain-receptors in the joint. In osteoarthrosis we encounter many destructive cases with radiological appearances almost identical with those of the Charcot hip joint, but in the absence of neuropathic causes we have operated as successfully as in ordinary cases of osteoarthrosis.

\section{Rheumatoid arthritis}

The operation may be performed at any time and even when the erythrocyte sedimentation rate is over $100 \mathrm{~mm} . / 1 \mathrm{st} \mathrm{hr}$.

Osteoporosis is no contraindication, because the body weight is diffused over a large area of bone through the medium of acrylic cement.

\section{Ankylosing spondylitis}

Complete ankylosis of the hip joint is no contraindication; indeed we have some of our most spectacular results in this condition especially when the patient's knees are unaffected.

\section{Polyarthritis}

The average age of patients is lower than that of those with osteoarthrosis; we frequently do bilateral hip replacement in Still's disease in patients of 16 to 18 years of age and in rheumatoid arthritis in the 20 s and 30s. A useful test in these cases is to determine whether or not there exists some element of what I have called 'Built-in Restraint' which, after hip surgery will persist to hold back the patient's function below the equivalent for a normal person 
of that age. 'Built-in Restraint' in the poly-arthritic lies usually in the knees and ankles, but sometimes necrobiotic nodules and sero-positive disease indicate a limited prognosis for survival. Because pain in the hip joints is often the dominating feature of the clinical state in patients with polyarthritis, it is possible to confer a great boon by total hip replacement even though they may still be well below normal function for their age. In these cases the artificial hips are likely to last indefinitely.

In patients between 20 and 40 years of age with local hip conditions, without systemic disease, the indications for total hip replacement are difficult to define at the present moment. The commonest examples are seen in cases of congenital dysplasia of the hip joint and the late results of traffic accidents. In young patients there will be no 'Built-in Restraint' after hip surgery; the artificial joint will be faced with perhaps 30 years of mechanical violence even before the patient has reached 60 years of age. These joints will be subjected to such an intensity of violence over a period of time so much greater than anything we have so far encountered that it is unsafe to extrapolate from known experience.

In young persons in whom no 'Built-in Restraint' will exist after operation, it is sometimes helpful to apply what I call the 'Pseudarthrosis Test'. If a hip condition is such that a primary resection of the head and neck would confer benefit on the patient, then total hip replacement is absolutely indicated. This is because, were it to fail, and were it to prove impossible to replace it by a second repair, then removing the implant would leave the patient better, or at least not worse, than in his original condition. If, on the other hand, one would not dream of performing a pseudarthrosis as a primary measure, then the utmost caution should be exercised in considering total hip replacement.

In this context the old surgical maxim in hip surgery still applies that operation is usually to be avoided unless undertaken for pain as the primary symptom. It is profoundly to be hoped that some day we shall be able to offer total hip replacements to young women, usually with congenital dislocation, whose main motive is not so much to relieve their pain as to improve their appearance. On the other hand improved neo-natal care will reduce the number of these difficult problems even within this decade.

\section{Centres for special surgery}

I should like to finish this account of the current status of total hip replacement with some remarks on the organization of services for prosthetic joint replacement. Even without considering knee joints or elbow joints, the work-load for total hip replacement is formidable and no provision for its ad- ministration has been made for it in the British National Health Service of the future. My experience during the last 5 years of intense specialization in this area shows that, only by grouping surgical expertise and equipment, can the maximum number of patients receive maximum quality. Nevertheless, there is currently no sign of any trend towards copying the pattern established at Wrightington. Perhaps I am blind to defects of extreme specialization which may be obvious to others; but, on the other hand, perhaps for others the pressure of day-to-day work is obscuring trends which will soon require decisive action.

One of these trends, clearly visible at Wrightington, is the changing attitude of patients to the medical profession. In acute emergencies patients have no alternative but to accept the medical services immediately at hand, whatever may be their natural inclination, but in cases of chronic disease and in elective surgery matters are very different, especially when we consider a disease which is relatively common. Patients from different parts of the country, and even of the world, discuss their problems over periods of years, and they meet patients who have had similar operations, with the result that they arrive at their own decisions. The trend in modern times is to seek specialist opinion for everything from motor cars to medicine. This is especially relevant in total hip replacement, because here it is obvious to the patient and his friends which are first class results and which are second class. In many areas of surgery the quality of a surgical result cannot be judged by patients or relatives; patients often have to ask their medical advisers whether the result is to be regarded as good or not, and if it is said to be good the patient has to be satisfied.

Although I advocate special centres for this type of surgery, so many of these operations will be required each year that it is impossible to insist that all should be done in special centres. It would not be unreasonable, however, to plan so that perhaps half the work is done in special centres; in this way permanent groups of consultants with special experience in this field could become available: first for patients offering special technical problems; secondly for the teaching of technical surgery to post-graduates who will handle this work in general hospitals, and thirdly to form an unbroken line of 'know-how' to cope with total joint replacements which may go into mechanical failure after 20 years in the body. To countenance the insertion of a total hip replacement into a patient of 25 years of age in 1971, without having a service station planned and organized for 1996, is like selling motor cars without providing mechanics and workshops.

When one tries to consider how highly specialized techniques can best be made available to large numbers of patients in the community, one has to 
face the fact that it is impossible, now or at any time in the future, and even in the most wealthy countries, to avoid some type of rationing (which Enoch Powell had described by the curious phrase 'rationing at the point of consumption'). Rationing will have to be a product of educating surgeons in methods of assessing priority for surgery, and of self-discipline in patients fostered by education. Uniform criteria of priority for surgery can be established in large centres in a way which is not possible in small centres. The most dangerous unit is the small unit looking for work. By having large centres with standardized criteria, graded waiting lists can be assembled so that the less disabled do not postpone the treatment of the grossly disabled as happens if all take their chronological turn on one waiting list. The quality of a surgical unit derives just as much from surgery which is not performed as from that which is well performed.
The future of hospital waiting lists in total joint replacement will present some difficult moral problems, rather like the priorities for renal dialysis. The chief problem will be the rival claims with the knee and elbow replacements, because these are fields where I think inventive enthusiasm may obsoure the usefulness of the result. Total knee and elbow replacements have their main indications in the polyarthritic, but the situation may arise in which one patient with polyarthritis may absorb surgical time and nursing care equivalent to six hip arthroplasties and may still not be self-supporting in the end. This emphasizes the importance of research to find the cause of rheumatoid arthritis in the hope of preventing it. Total joint replacement may eventually be used only in mechanical diseases of the joints, though I for one believe that the arthrosic hip, having a genetic basis which can never be altered, will always come into this category. 